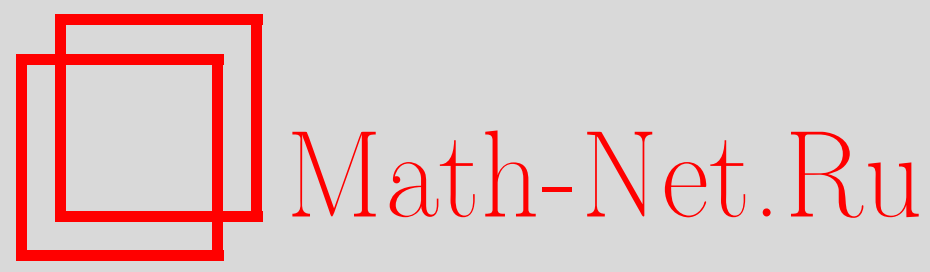

Р. В. Галиулин, С. Н. Михалёв, И. Х. Сабитов, Некоторые приложения формулы для объема октаэдра, Матем. заметки, 2004, том 76, выпуск 1, 27-43

DOI: https://doi.org/10.4213/mzm88

Использование Общероссийского математического портала Math-Net.Ru подразумевает, что вы прочитали и согласны с пользовательским соглашением http://www.mathnet.ru/rus/agreement

Параметры загрузки:

IP : 3.93.64.190

26 апреля 2023 г., 13:43:44 


\title{
НЕКОТОРЫЕ ПРИЛОЖЕНИЯ ФОРМУЛЫ ДЛЯ ОБЪЕМА ОКТАЭДРА
}

\author{
Р.В. Галиулин, С.Н. Михалёв, И.Х. Сабитов
}

\begin{abstract}
Вводятся понятия комбинаторной, метрической и пространственной симметрий многогранника и в случае симметричных октаэдров приводятся явные виды канонических многочленов для объемов.

Библиография: 3 названия.
\end{abstract}

1. Введение. В [1] доказано, что для каждого многогранника $P$ в $\mathbb{R}^{3}$ с треугольными гранями существует многочлен $Q(V)$ такой, что его коэффициенты определяются только комбинаторным строением $P$ и полиномиально зависят от квадратов длин его ребер (с общим обозначением $l^{2}$ ), а объем многогранника является корнем этого многочлена. В [2] предлагается способ нахождения канонического (т.е. имеющего наименьшую возможную степень) многочлена для объема $V$ октаэдра. Этот многочлен имеет вид

$$
Q(V)=V^{16}+a_{1}\left(l^{2}\right) V^{14}+\cdots+a_{8}\left(l^{2}\right) .
$$

Каждое слагаемое $a_{i}\left(l^{2}\right) V^{16-2 i}, 1 \leqslant i \leqslant 8$, многочлена (1) имеет одну и ту же размерность $\mid$ см $\left.\right|^{48}$, и поскольку в него, кроме объема, входят еще 12 переменных длин ребер, то количество мономов оказьвается чрезвычайно большим (согласно оценке из [2] оно порядка $10^{10}$ ), поэтому реально даже на мощном компьютере такой полином вычислен быть не может. С другой стороны, в некоторых частных случаях (например, когда имеется достаточно много равных ребер) вычисление этого многочлена оказывается вполне возможным. Такая ситуация имеет место, например, в кристаллографии, где работают с октаэдрами, имеющими некоторую симметрию.

В данной работе вводятся различные понятия симметрии многогранника, и для большинства симметричных октаэдров вычисляется канонический многочлен для его объeмa.

2. Три вида симметрии многогранников. Пусть дан некоторьй симплициальный комплекс $K$ с $n$ вершинами. Мы скажем, что симплициальньй комплекс $K$ имеет комбинаторную симметрию, если существует автоморфизм множества его вершин, сохраняющий инцидентность вершин и ребер. Любой такой автоморфизм мы будем назьвать комбинаторным преобразованием симметрии. Очевидно, что комбинаторные преобразования симметрии комплекса $K$ образуют группу относительно операции

Работа первого автора выполнена при поддержке Российского фонда фундаментальных исследований, грант № 99-01-00867; работа второго и третьего авторов выполнена при поддержке фонда ИНТАС-РФФИ, грант № IR-97-01-71010. 
композиции. Далее, пусть ребрам симплициального комплекса $K$ приписаны некоторые значения длин (с выполнением неравенства треугольника на гранях). Мы скажем, что полученньй метрический симплициальньй комплекс обладает метрической симметрией, если существует комбинаторное преобразование симметрии, сохраняющее длины ребер. Если $G_{k}$ - групша комбинаторной симметрии, $G_{m}$ - групша метрической симметрии, то $G_{m} \subset G_{k}$. Пусть отображение $P: K \rightarrow \mathbb{R}^{3}$ реализует метрический сиплициальньй комплекс $K$ в виде некоторого многогранника $P(K)$ в $\mathbb{R}^{3}$. Мы скажем, что комбинаторно симметричньй симплициальный комплекс $K$ имеет реализацию в $\mathbb{R}^{3}$ в виде многогранника с пространственной симметрией, если существует движение (первого или второго рода), переводящее этот многогранник в себя.

Очевидно, что многогранник с пространственной симметрией является и комбинаторно, и метрически симметричным. Обратное, вообще говоря, неверно: реализация метрически симметричного симплициального комплекса $K$ в виде многогранника в $\mathbb{R}^{3}$ может не иметь никакой нетривиальной пространственной симметрии. Соответствующий пример строится так: рассмотрим вьпуклый октаэдр $P$ в $\mathbb{R}^{3}$ с плоским экватором, разделяющим $P$ на две части $P^{\prime}$ и $P^{\prime \prime}$, симметричные относительно плоскости экватора. Пусть $P$ не имеет никакой другой пространственной симметрии. Рассмотрим теперь другой октаэдр $P_{1}$, получаемьй из $P$ отражением относительно плоскости симметрии части $P^{\prime \prime}$. Октаэдр $P_{1}$, таким образом, совпадает с дважды покрытой частью $P^{\prime}$ исходного октаэдра $P$. Метрическая симметрия октаэдров $P$ и $P_{1}$ одинакова, поскольку эти октаэдры изометричны (являются пространственными реализациями одного и того же метрического симплициального комплекса), однако октаэдр $P_{1}$ не имеет никакой нетривиальной пространственной симметрии. Ниже мы покажем, что может существовать реализация, у которой группа пространственных симметрий нетривиальна, но меньше, чем группа метрической симметрии. Кроме того, можно сформулировать следующие нерешенные задачи.

1) Пусть метрически симметричный октаэдр допускает некоторую изометрическую реализацию в $\mathbb{R}^{3}$; обязательно ли найдется реализация без пространственной симметриии?

2) Существует ли метрически симметричный октаэдр, для которого никакая его изометрическая реализация в $\mathbb{R}^{3}$ не имеет пространственной симметрии?

3. Симметрии октаэдра. 1) Комбинаторная симметрия. Рассмотрим биективные отображения множества вершин октаэдра в себя такие, что сохраняется инцидентность вершин и ребер. Пусть вершины октаэдра пронумерованы от 1 до 6 как на рис. 1. Тогда любое такое отображение удобно рассматривать как некоторую перестановку

$$
\sigma=\left(\begin{array}{cccccc}
1 & 2 & 3 & 4 & 5 & 6 \\
i_{1} & i_{2} & i_{3} & i_{4} & i_{5} & i_{6}
\end{array}\right)
$$

на множестве вершин. Очевидно, рассматриваемые отображения образуют группу $G_{k}$, изоморфную некоторой подгрупше группы перестановок $S_{6}$. Оказывается, порядок $G_{k}$ равен 48. В этом легко убедиться следующим образом. Любая перестановка $\sigma \subset G_{k}$ должна сохранять также и неинцидентность вершин и ребер: если две вершины не соединены ребром, то их образы также не должны быть соединены ребром. Значит, каждая из пар вершин $(1,6),(2,4),(3,5)$ может перейти только в одну из пар $(1,6),(2,4),(3,5)$, причем разныепары переходят в разные пары; таким образом, имеется $6=3$ ! вариантов для пар вершин. Кроме того, для каждой из трех пар вершин точки $(i, j)$ могут перейти как в $(k, l)$, так и в $(l, k)$. Значит, внутри каждого из 6 случаев имеем еще $2^{3}=8$ вариантов. Всего, таким образом, в групе $G_{k}$ получается не более $6 \cdot 8=48$ элементов. 


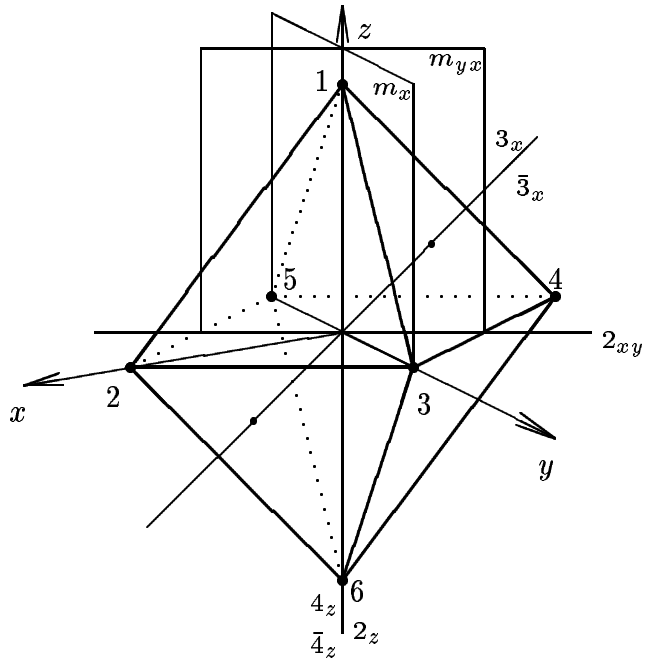

Рис. 1

Простой проверкой легко убедиться, что все эти перестановки различны, лишних среди них нет, и порядок группы $G_{k}$, таким образом, действительно равен 48 .

2) Метрическая симметрия. Требование, чтобы данный октаэдр обладал метрической симметрией $G_{m}$, является ограничением на его метрику: некоторые ребра должны иметь одинаковую длину. В частности, октаэдр, у которого все ребра различны, не имеет метрической симметрии (т.е. он обладает лишь тривиальной группй метрической симметрии). Для правильного октаэдра (все ребра которого равны между собой) группа метрической симметрии совпадает, очевидно, с группой $G_{k}$ комбинаторной симметрии октаэдра.

3) Пространственная симметрия. Пусть теперь мы имеем некоторую конкретную реализацию октаэдра в $\mathbb{R}^{3}$. В случае, если у октаэдра имеется центр, (инверсионная) ось, или плоскость симметрии, мы говорим, что октаэдр имеет соответствующую пространственную симметрию. Принятые в кристаллографии обозначения элементов симметрии и соответствующих им преобразований симметрии приведены в таблице 1.

ТАБЛИЦА 1

\begin{tabular}{|c|c|c|}
\hline элемент симметрии & преобразование симметрии & обозначение \\
\hline \hline ось симметрии 4-го порядка & поворот на $\pi / 2$ вокруг оси & 4 \\
\hline ось симметрии 3-го порядка & поворот на $2 \pi / 3$ вокруг оси & 3 \\
\hline ось симметрии 2-го порядка & поворот на $\pi$ вокруг оси & 2 \\
\hline $\begin{array}{c}\text { инверсионная ось симметрии } \\
\text { 4-го порядка }\end{array}$ & $\begin{array}{c}\text { композиция поворота на } \pi / 2 \\
\text { вокруг оси и инверсии относительно } \\
\text { точки на оси }\end{array}$ & $\overline{4}$ \\
\hline $\begin{array}{c}\text { инверсионная ось симметрии } \\
\text { 3-го порядка }\end{array}$ & $\begin{array}{c}\text { композиция поворота на } 2 \pi / 3 \\
\text { вокруг оси и инверсии относительно } \\
\text { точки на оси }\end{array}$ & $\overline{3}$ \\
\hline центр симметрии & симметрия относительно центра & $i$ \\
\hline плоскость симметрии & отражение относительно плоскости & $m$ \\
\hline
\end{tabular}


Различают диагональные и координатные оси симметрии. Ось симметрии называется координатной в том случае, когда она совпадает с одной из осей координат, и диагональной, если она является бисектриссой плоского угла между какими-то двумя осями координат. Аналогично, плоскости симметрии могут быть диагональными и координатньпи.

Очевидно, что любому пространственному преобразованию симметрии соответствует некоторый автоморфизм $\sigma \subset G_{k}$; например, для элементов симметрии на рис. 1 получаем

$$
\begin{array}{rlrl}
4_{z} & \leftrightarrow\left(\begin{array}{llllll}
1 & 2 & 3 & 4 & 5 & 6 \\
1 & 3 & 4 & 5 & 2 & 6
\end{array}\right), & \overline{4}_{z} \leftrightarrow\left(\begin{array}{llllll}
1 & 2 & 3 & 4 & 5 & 6 \\
6 & 5 & 2 & 3 & 4 & 1
\end{array}\right), \\
3_{x} & \leftrightarrow\left(\begin{array}{lllllll}
1 & 2 & 3 & 4 & 5 & 6 \\
3 & 6 & 4 & 1 & 2 & 5
\end{array}\right), & \overline{3}_{x} \leftrightarrow\left(\begin{array}{llllll}
1 & 2 & 3 & 4 & 5 & 6 \\
5 & 1 & 2 & 6 & 4 & 3
\end{array}\right), \\
2_{z} & \leftrightarrow\left(\begin{array}{llllll}
1 & 2 & 3 & 4 & 5 & 6 \\
1 & 4 & 5 & 2 & 3 & 6
\end{array}\right), & 2_{x y} \leftrightarrow\left(\begin{array}{llllll}
1 & 2 & 3 & 4 & 5 & 6 \\
6 & 5 & 4 & 3 & 2 & 1
\end{array}\right), \\
m_{x} & \leftrightarrow\left(\begin{array}{llllll}
1 & 2 & 3 & 4 & 5 & 6 \\
1 & 4 & 3 & 2 & 5 & 6
\end{array}\right), & m_{y x} \leftrightarrow\left(\begin{array}{llllll}
1 & 2 & 3 & 4 & 5 & 6 \\
1 & 5 & 4 & 3 & 2 & 6
\end{array}\right), \\
i & \leftrightarrow\left(\begin{array}{llllll}
1 & 2 & 3 & 4 & 5 & 6 \\
6 & 4 & 5 & 2 & 3 & 1
\end{array}\right) . & &
\end{array}
$$

УТВЕРЖДЕНИЕ. Группа $G$ пространственной симметрии правильного октаэдра изоморфна әруппе $G_{k}$ комбинаторной симметрии октаэдра.

ДокАЗАтЕЛьСтво. Установим изоморфизм $f: G \rightarrow G_{k}$. Для этого рассмотрим правильньй октаэдр, стандартным образом расположенный в $\mathbb{R}^{3}$ (см. рис. 1 ). Всякое пространственное преобразование симметрии $s \in G$ по определению переводит октаэдр в себя, а значит, индуцирует некоторую перестановку $\sigma \in S_{6}$ множества вершин, причем $\sigma \in G_{k} \subset S_{6}$, т.е. сохраняется отношение инцидентности вершин и ребер. Положим $f(s)=\sigma$. Очевидно, что отображение $f$ сохраняет операцию композиции. Докажем, что отображение $f$ инъективно. Действительно, предположим, что два различных пространственных преобразования симметрии октаэдра $s_{1}, s_{2} \in G, s_{1} \neq s_{2}$, индуцируют одну и ту же перестановку $\sigma \in G_{k}$. Тогда $f\left(s_{1}^{-1} \cdot s_{2}\right)=f\left(s_{1}^{-1}\right) \cdot f\left(s_{2}\right)=\sigma^{-1} \cdot \sigma=e$, где $e \in S_{6}$ - тождественная перестановка, и пространственное преобразование $s_{1}^{-1} \cdot s_{2}$ оставляет неподвижными все 6 вершин октаэдра, среди которых, конечно, найдутся 4 точки, не лежащие на одной плоскости. А линейное преобразование пространства $\mathbb{R}^{3}$, имеющее хотя бы 4 неподвижные точки, не лежащие на одной плоскости, является тождественньм, поэтому тождественным преобразованием является и $s_{1}^{-1} \cdot s_{2}$, что невозможно для различньх пространственных симметрий $s_{1}$ и $s_{2}$ октаэдра.

Построенное инъективное и сохраняющее групповую операцию отображение $f: G$ $\rightarrow G_{k}$ является изоморфизмом, поскольку мощности множеств $G$ и $G_{k}$ совпадают: из кристаллографии известно (см. [3]), что группа пространственной симметрии правильного октаэдра имеет порядок 48 , т.е. $|G|=\left|G_{k}\right|=48$.

4. Многочлены для объемов метрически симметричных октаэдров. Как уже было сказано, количество мономов многочлена для объема (1) уменьшается в случае, если некоторые ребра октаэдра имеют одинаковую длину. Поэтому при вычислении многочлена для объема метрически симметричных октаэдров можно ожидать существенных упрощений. 
Поступаем следуюшим образом. Перебираем все подгрупшы групшы $G$ пространственных симметрий правильного октаэдра (полньй список этих подгрупп позаимствован из кристаллографии, см. [3]). Для каждой подгрупшы $G^{\prime} \subset G$ берем соответствующую ей при построенном вьше изоморфизме $f: G \rightarrow G_{k}$ группу $G_{k}^{\prime} \subset G_{k}$ автоморфизмов на множестве вершин октаэдра. Полагаем групу $G_{k}^{\prime}$ групой метрической симметрии октаэдра. Вьписываем соответствующее ограничение на метрику и вычисляем многочлен $Q(V)$ для объема соответствующего метрически симметричного октаэдра, используя предложенньй в работе [2] способ.

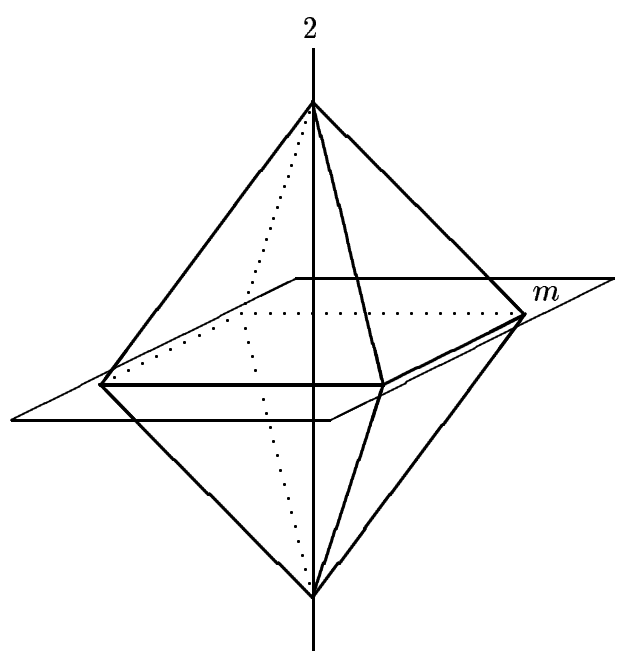

Рис. 2

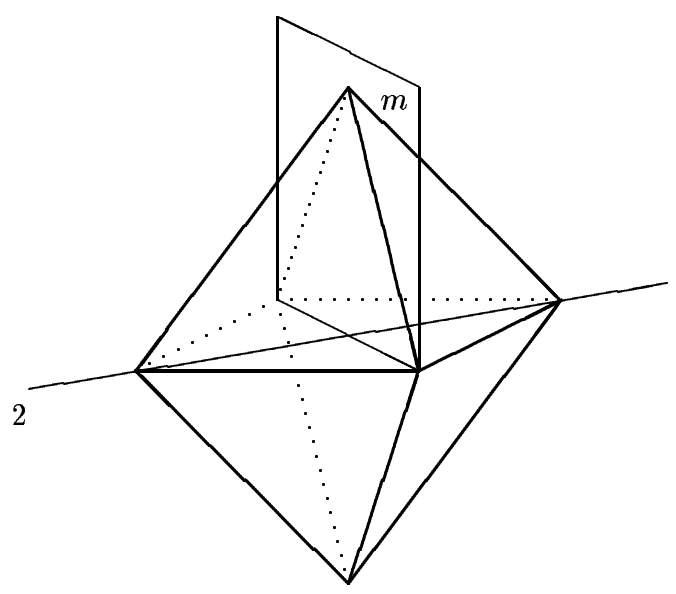

Рис. 3

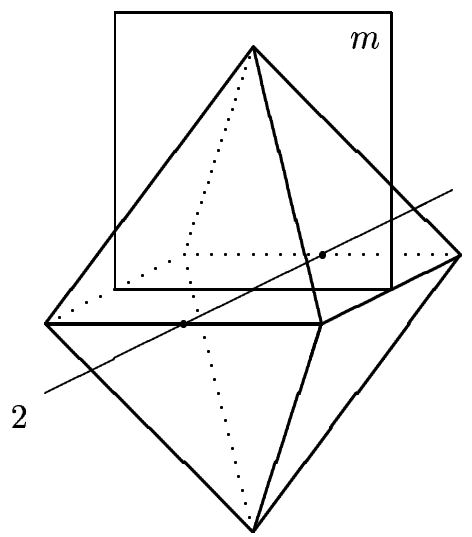

Рис. 4

Построение многочлена $Q(V)$ для произвольного октаэдра основано на двух фактах (см. [2]). Во-первых, существует явная формула для вычисления квадрата объема октаэдра по длинам его ребер и диагоналей (в нашей задаче диагонали неизвестны). Во-вторых, для произвольной шестерки точек в $\mathbb{R}^{3}$ можно вьписать некоторые полино- 
миальные условия, которым удовлетворяют попарные расстояния между этими точками, т.е. длины ребер и диагоналей октаэдра не являются независимыми. Эти два обстоятельства позволили авторам работы [2] составить некоторую матрицу $S$ порядка $8 \times 8$, элементы которой являются многочленами от длин ребер октаэдра, такую, что квадрат объема соответствуюшего октаэдра является собственньм значением этой матрицы, т.е. корнем собственного многочлена $Q(V)$ матрицы $S$. Многочлен $Q(V)$, старший коэф фициент которого равен 1 , имеет степень 8 и является минимальным по степени многочленом для объема октаэдра.

При вычислении многочленов $Q(V)$ для метрически симметричных октаэдров мы учитываем соответствующие равенства ребер еще в процессе формирования матрицы $S$ и только после этого, уже для существенно упрощенной матрицы, вычисляем ее собственньй многочлен, которьй и является минимальным многочленом для объема октаэдра с данной метрической симметрией.

\section{ТАБЛИЦА 2}

\begin{tabular}{|c|l|}
\hline 48 & $m 3 m$ (полная группа пространственной симметрии) \\
\hline 24 & $432, \overline{4} 3 m, m 3$ \\
\hline 16 & $4 / m m m$ \\
\hline 12 & $23, \overline{3} m$ \\
\hline 8 & $422,4 m m, m m m, \overline{4} m 2, \overline{4} 2 m, 4 / m$ \\
\hline 6 & $312,3 m, \overline{3}$ \\
\hline 4 & $2 / m, m m 2,222,4, \overline{4}$ \\
\hline 3 & 3 \\
\hline 2 & $i, 2, m$ \\
\hline
\end{tabular}

ЗАмЕчАнИЕ 1. Имеет смысл рассматривать только существенно различныеподгруппы групшы $G$ и лишь для них вычислять многочлены. Имеется в виду следуюшее. Предположим, дан октаэдр в $\mathbb{R}^{3}$, имеющий некоторую пространственную симметрию, например, координатную ось симметрии 2-го порядка, и перпендикулярную ей координатную плоскость симметрии (группа $2 \mid m$, см. рис. 2$)$. Этой комбинаторной подгруппе $G_{k}^{1} \subset G_{k}$ соответствует некоторая метрическая симметрия октаэдра. Но в качестве элементов симметрии можно выбрать другую координатную ось и перпендикулярную этой оси координатную плоскость симметрии (см. рис. 3). Группа $G_{k}^{2} \subset G_{k}$ комбинаторной симметрии при этом будет изоморфной $G_{k}^{1}: G_{k}^{1} \cong G_{k}^{2}$. Метрическая же симметрия изменится; стало быть, изменится и формула для объема. Однако изменения формулы в этом случае сводятся, очевидно, к переобозначению переменных. С такой точки зрения, символ $2 \mid m$ обозначает цельй класс подгрупп групшы $G_{k} \cong G$, приводящих к одинаковым с точностью до переобозначения переменных формулам. Изоморфные подгрупшы $G_{k}^{1}$ и $G_{k}^{2}$ представлены наборами элементов симметрии одинаковьх типов (координатная ось 2-го порядка и перпендикулярная ей координатная плоскость). Иначе говоря, во втором случае октаэдр можно расположить по-другому так, что элементы его симметрии будут теми же, что и в первом его расположении.

Наоборот, если мы выберем в качестве элементов симметрии перпендикулярные друг другу диагональную ось симметрии 2-го порядка и диагональную плоскость симметрии (рис. 4$)$, то несмотря на то что группа $G_{k}^{3} \subset G_{k}$ будет по-прежнему изоморфна группе $G_{k}^{1}: G_{k}^{3} \cong G_{k}^{1}$, порождающие их элементы симметрии имеют разньй тип (диагональные и координатные соответственно). Формулы для объемов для случаев подгруш 
$G_{k}^{3}$ и $G_{k}^{1}$ отличаются существенно.

Перейдем теперь к перечислению подгрупп группы $G$ с использованием принятых в кристаллографии обозначений. Как правило, символы, фигурирующие в обозначении подгрупшы, соответствуют ее образующим. Для каждой подгруппы на чертеже изображены элементы симметрии, соответствующие ее образующим; для большинства подгрупп вьписан полученньй многочлен для объема соответствующего метрически симметричного октаэдра.

По теореме Лагранжа порядки подгрупп групшы $G$ пространственной симметрии октаэдра могут быть следующими: $24,16,12,8,6,4,3,2,1$. В таблице 2 представлены все различные подгрупшы группы $G$ (см. [3]).

Для экономии места мы в простых случаях вместо рисунка даем его словесное описание со ссылкой на следующую модель октаэдра (рис. 5).

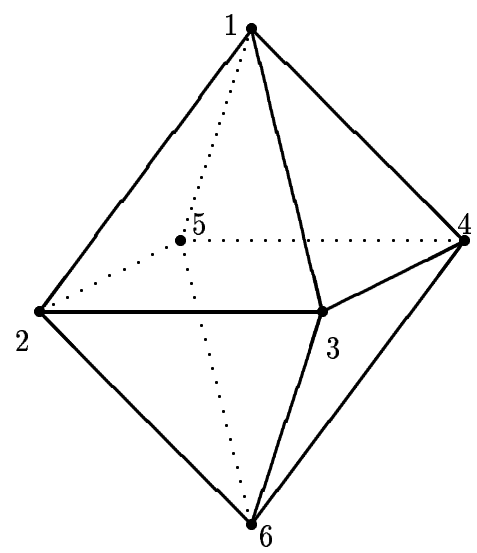

Рис. 5

Начнем со случая, когда группа метрической симметрии совпадает со всей группой $G_{k}$ комбинаторной симметрии, т.е. рассмотрим подгруппу $m 3 m$. В этом случае метрическая симметрия максимальна: все ребра октаэдра равны между собой, т.е.

$$
l_{12}=l_{13}=l_{14}=l_{15}=l_{23}=l_{34}=l_{45}=l_{52}=l_{62}=l_{63}=l_{64}=l_{65}=a \text {. }
$$

Тогда многочлен $Q(v)$ для объема имеет вид

$$
Q(v)=v^{8}-8 A^{3} v^{7}
$$

причем выражение для положительного корня уравнения $Q(v)=0$ представляет собой известную формулу для объема правильного октаэдра.

Здесь и далее $v=36 V^{2}$, где $V$ - объем октаэдра. Кроме того, мы будем придерживаться следуюшего соглашения: если длина некоторого ребра обозначена строчной латинской буквой, то квадрат длины этого ребра обозначается соответствующей заглавной буквой; так например, здесь $A=a^{2}$. 
Переходим к подгруппам с меньшим числом элементов.

С точностью до изоморфизма в $G$ имеется 3 подгруппы 24 -го порядка: $432, \overline{4} 3 m$ и $m 3$. Оказывается, что и в каждом из этих случаев метрическая симметрия максимальна (все ребра равны между собой), поэтому одинаковыми будут и формулы для объемов. Таким образом, абстрактньй октаэдр, группй метрической симметрии которого является одна из групп 24 -го порядка $(432, \overline{4} 3 m$ или $m 3)$, автоматически имеет и большую метрическую симметрию, а именно, группой его метрической симметрии является вся группа $m 3 m$. Тем же свойством обладают и октаэдры, имеющие в качестве групш метрической симметрии группы 23 и 312 (см. ниже случаи 6) и 14)). Случай подгруппы 312 интересен по следующим причинам. Метрическая симметрия октаэдра по-прежнему максимальна - все ребра равны между собой. Но, оказьвается, в $\mathbb{R}^{3}$ существует изометрическая реализация абстрактного октаэдра со всеми равными ребрами такая, что группой пространственной симметрии получаемого многогранника является в точности группа 312. Такой реализацией является восьмикратно покрытьй равносторонний треугольник. Легко убедиться, что эта реализация обладает требуемьми свойствами.

Итак, приступаем к перечислению неизоморфных подгрупп группы $m 3 \mathrm{~m}$.

2) 432 - в этом случае образующими ${ }^{1}$ являются ось симметрии 4-го порядка (проходящая через вершины 1 и 6) и ось симметрии 3-го порядка (проходящая через центры граней 134 и 256), а метрическая симметрия максимальна:

$$
l_{12}=l_{13}=l_{14}=l_{15}=l_{23}=l_{34}=l_{45}=l_{52}=l_{62}=l_{63}=l_{64}=l_{65}=a \text {. }
$$

Многочлен для объема имеет следующий вид:

$$
Q(v)=v^{8}-8 A^{3} v^{7} .
$$

В случаях 3) и 4) (и некоторых других, см. далее) метрическая симметрия также оказьвается максимальной, поэтому многочлен $Q(V)$ получается такой же как в случае 2 ) (подгруппа 432)

3) $\overline{4} 3 m$ - образующими являются инверсионная ось симметрии 4-го порядка (проходящая через вершины 1 и 6) и ось симметрии 3-го порядка (проходящая через центры граней 134 и 256),

$$
Q(v)=v^{8}-8 A^{3} v^{7} .
$$

4) $m 3$ - в этом случае см. рис. 6 и

$$
Q(v)=v^{8}-8 A^{3} v^{7} .
$$

5) $4 / m m m$ - с точностью до изоморфизма это единственная подгруппа 16-го порядка. В этом случае см. рис. 7 и многочлен для объема октаэдра с такой групой симметрии имеет следующее выражение:

$$
Q(v)=v^{8}+\left(8 B^{3}-16 B^{2} A\right) v^{7} .
$$

В группе $G$ имеется две неизоморфные подгруппы 12 -го порядка: 23 и $\overline{3} m$.

6) 23 - образующими являются ось симметрии 2-го порядка (проходящая через вершины 3 и 5) и ось симметрии 3-го порядка (проходящая через центры граней 134 и 256),

\footnotetext{
${ }^{1}$ Здесь и далее мы позволяем себе некоторую вольность речи: на самом деле образующими группы преобразований являются, кончено, не сами геометрические элементы симметриии (оси и плоскости), а преобразования симметрии относительно этих элементов (вращения и отражения).
} 


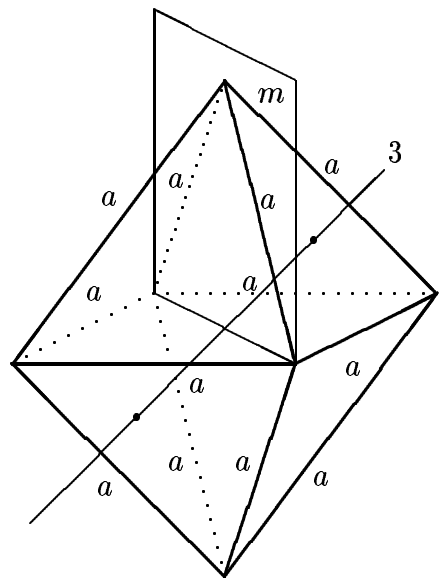

Рис. 6

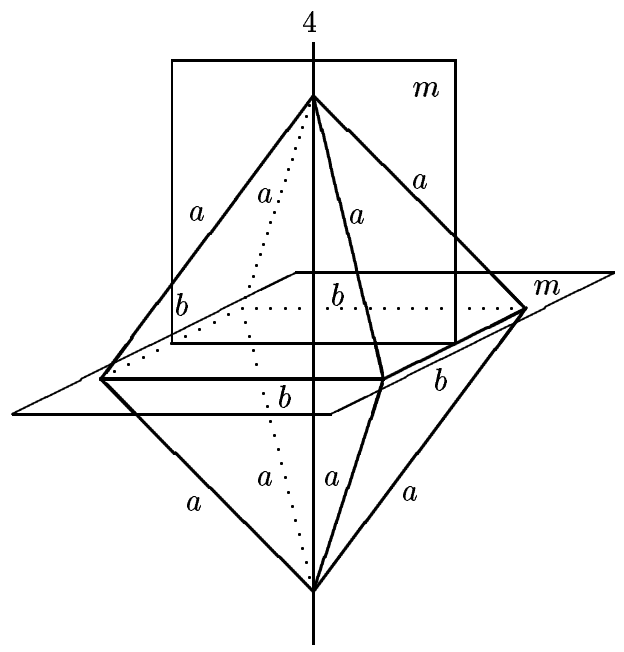

Рис. 7

а метрическая симметрия максимальна; следовательно, многочлен $Q(V)$ такой же как в случае 2) (подгруппа 432):

$$
Q(v)=v^{8}-8 A^{3} v^{7}
$$

7) $\overline{3} m-$ в этом случае см. рис. 8 и

$$
Q(v)=v^{8}+\left(4 A^{3}-12 C A^{2}\right) v^{7}
$$

В $G$ имеется 6 различньх подгрупп 8-го порядка: $422,4 m m, m m m, \overline{4} m 2, \overline{4} 2 m, 4 / m$.

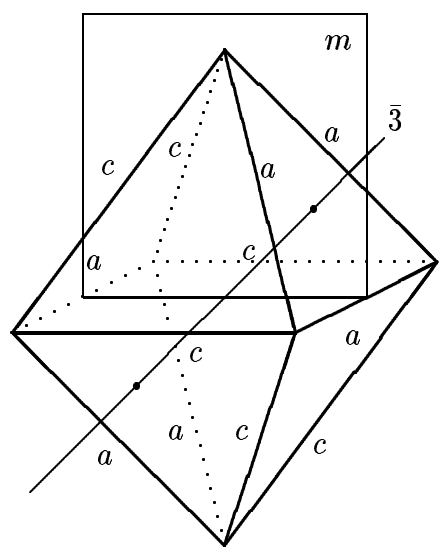

Рис. 8

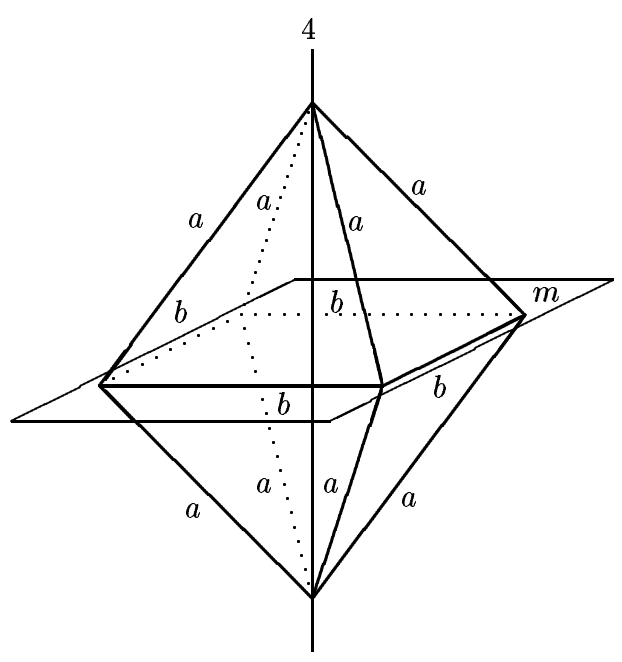

Рис. 9 
8) 422 - в этом случае образуюшими являются ось симметрии 4-го порядка (проходящая через вершины 1 и 6) и диагональная ось симметрии 2-го порядка (проходящая через середины ребер 23 и 45 ),

$$
l_{12}=l_{13}=l_{14}=l_{15}=l_{62}=l_{63}=l_{64}=l_{65}=a, \quad l_{23}=l_{34}=l_{45}=l_{52}=b
$$

(в этом случае октаэдр автоматически обладает и большей группой метрической симметрии, а именно, $4 / \mathrm{mmm})$. Многочлен для объема имеет следующий вид:

$$
Q(v)=v^{8}+\left(8 B^{3}-16 B^{2} A\right) v^{7} .
$$

9) $4 \mid m$ - этот случай аналогично предыдущему также сводится к случаю $4 / m m m$ (см. рис. 9) и

$$
Q(v)=v^{8}+\left(8 B^{3}-16 B^{2} A\right) v^{7} .
$$

10) $4 m m$ - в этом случае см. рис. 10 и

$$
Q(v)=v^{8}+\left(-8 A C^{2}+8 C^{3}-8 B C^{2}\right) v^{7}+\left(16 B^{2} C^{4}-32 C^{4} B A+16 C^{4} A^{2}\right) v^{6} .
$$

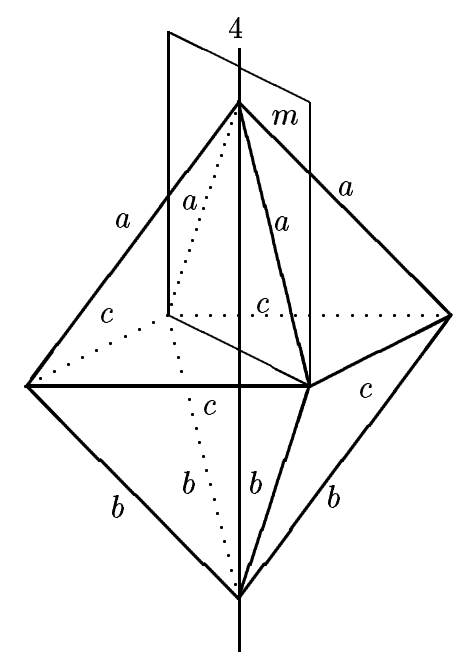

Рис. 10

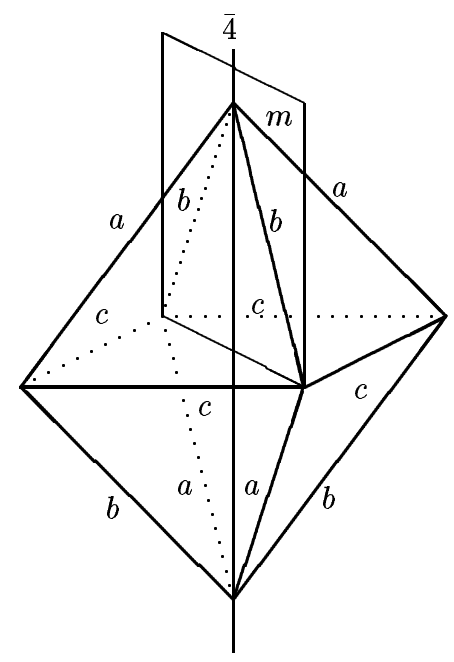

Рис. 11

11) $\overline{4} m 2$ - в этом случае см. рис. 11 и

$$
\begin{aligned}
Q(v)= & v^{8}+\left(-16 C B A-8 B C^{2}+8 B^{3}-8 B A^{2}+8 C A^{2}\right. \\
& \left.+8 C B^{2}+8 C^{3}-8 B^{2} A-8 A C^{2}+8 A^{3}\right) v^{7} \\
& +\left(128 B^{3} C A^{2}-64 B C^{3} A^{2}-16 A^{6}-192 B A^{4} C+128 A^{3} B^{2} C\right. \\
& +64 A^{3} C^{3}-240 A^{4} B^{2}+96 A^{5} B-96 A^{4} C^{2}+64 A^{5} C+128 B A^{3} C^{2} \\
& +320 A^{3} B^{3}-16 B^{6}-16 B^{2} C^{4}+64 B^{3} C^{3}-16 C^{4} A^{2}+64 C B^{5} \\
& -240 A^{2} B^{4}+32 C^{4} B A-192 C B^{4} A-64 C^{3} A B^{2}+128 C^{2} B^{3} A \\
& \left.-96 B^{4} C^{2}-64 B^{2} C^{2} A^{2}+96 A B^{5}\right) v^{6} .
\end{aligned}
$$




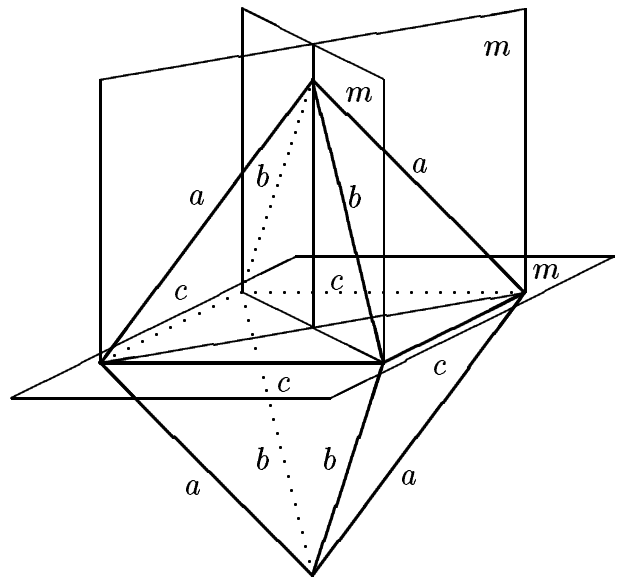

Рис. 12

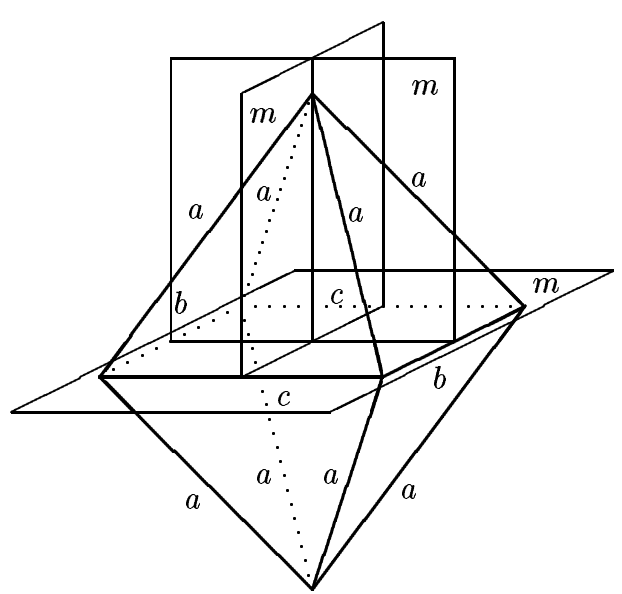

Рис. 13

Случай подгрупш $m m m$ имеет два подслучая.

12a) $m m m$ - образуюшие соответствуют трем координатным плоскостям (см. рис. 12) и

$$
\begin{aligned}
Q(v)= & v^{8}+\left(-8 B^{2} A+8 A^{3}+8 B^{3}-8 A C^{2}-8 C A^{2}\right. \\
& \left.+8 C^{3}+16 C B A-8 B A^{2}-8 C B^{2}-8 B C^{2}\right) v^{7}
\end{aligned}
$$

12b) $m m m$ - образующие соответствуют двум диагональным и одной координатной плоскостям симметрии (см. рис. 13) и

$$
Q(v)=v^{8}+\left(-16 C B A+4 B C^{2}+4 C B^{2}\right) v^{7} .
$$

13) $\overline{4} 2 m$ - в этом случае образующими являются инверсионная ось симметрии 4-го порядка (проходящая через вершины 1 и 6) и координатная ось симметрии 2-го порядка (проходящая через вершины 3 и 5 ), а метрическая симметрия и многочлен $Q(V)$ такие же, как в случае 5) (подгруппа $4 \mid \mathrm{mmm}$ ).

Переходим к подгрупшам порядка 6 (подгрупшы $312,3 m, \overline{3}$ ).

14) 312 - в этом случае образующими являются ось симметрии 3 -го порядка (проходящая через центры граней 134 и 256) и диагональная ось симметрии 2-го порядка (проходящая через середины ребер 34 и 25); метрическая симметрия максимальна; следовательно, многочлен $Q(V)$ такой же, как в случае 2) (подгруппа 432):

$$
Q(v)=v^{8}-8 A^{3} v^{7}
$$

15) $\overline{3}$ - в этом случае образующей является инверсионная ось симметрии 3-го порядка (проходящая через центры граней 134 и 256), а метрическая симметрия и, следовательно, многочлен $Q(V)$ такой же, как в случае 7 ) (подгруппа $\overline{3} m$ ):

$$
Q(v)=v^{8}+\left(4 A^{3}-12 C A^{2}\right) v^{7} .
$$




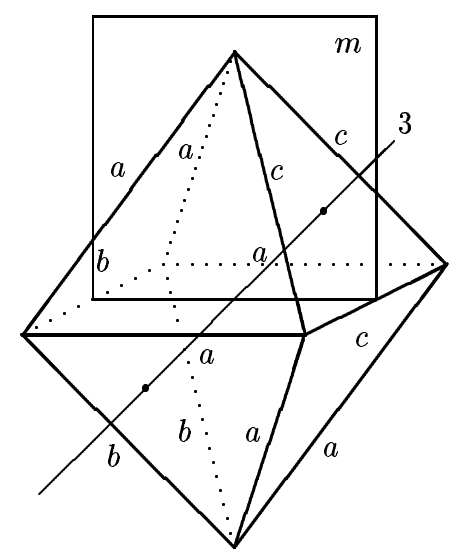

Рис. 14

16) $3 m$ - этот случай интересен тем, что, с одной стороны, все коэффициенты полинома $Q(v)$ оказьваются ненулевыми, a, с другой стороны, этот полином разлагается на множители; см. рис. 14 и

$$
\begin{aligned}
Q(v)= & \frac{1}{65536}\left(16 v^{2}+24 C B^{2} v+8 C^{3} v-144 C B A v+24 C^{2} B v-24 A C^{2} v\right. \\
& +8 B^{3} v-24 A B^{2} v+B^{6}-36 B C^{3} A^{2}+C^{6}-3 C B^{5}-36 B^{3} C A^{2} \\
& -2 B^{3} C^{3}+18 C B^{4} A-12 C^{3} A B^{2}+3 B^{2} C^{4}+18 C^{4} B A+54 B^{2} C^{2} A^{2} \\
& \left.+9 C^{4} A^{2}-12 C^{2} B^{3} A+3 B^{4} C^{2}+9 A^{2} B^{4}-3 C^{5} B-6 A C^{5}-6 A B^{5}\right) \\
\times & \left(16 v^{2}-8 C B^{2} v+8 C^{3} v+48 C B A v-8 C^{2} B v-24 A C^{2} v+8 B^{3} v\right. \\
& -24 A B^{2} v+B^{6}-36 B C^{3} A^{2}+C^{6}-3 C B^{5}-36 B^{3} C A^{2}-2 B^{3} C^{3} \\
& +18 C B^{4} A-12 C^{3} A B^{2}+3 B^{2} C^{4}+18 C^{4} B A+54 B^{2} C^{2} A^{2}+9 C^{4} A^{2} \\
& \left.-12 C^{2} B^{3} A+3 B^{4} C^{2}+9 A^{2} B^{4}-3 C^{5} B-6 A C^{5}-6 A B^{5}\right)^{3} .
\end{aligned}
$$

Подгруппы 4-го порядка имеют следующие обозначения: $2 / m, m m 2,222,4, \overline{4}$. При рассмотрении первьх трех из них будем выделять два подслучая.

17a) $2 \mid m$ - образующим соответствуют координатная ось симметрии и диагональная плоскость симметрии (см. рис. 15) и

$$
\begin{aligned}
Q(v)= & v^{8}+\left(4 C^{2} D-4 C B^{2}+8 C B A-8 C B D-4 C A^{2}-8 C A D+4 C D^{2}\right. \\
& \left.+8 B^{3}-8 B^{2} A-4 B^{2} D-8 B A^{2}+8 B A D+8 A^{3}-4 A^{2} D\right) v^{7} .
\end{aligned}
$$

17b) $2 \mid m$ - образующим соответствуют диагональная ось симметрии и координатная плоскость симметрии (см. рис. 16) и

$$
Q(v)=v^{8}+\left(4 C D^{2}-8 C B D-8 C A D+4 C^{2} D+4 A^{2} D-8 B A D+4 B^{2} D\right) v^{7} .
$$

18a) $m m 2$ - образующим соответствуют две диагональные плоскости симметрии (см. рис. 17) и

$$
\begin{aligned}
Q(v)= & v^{8}+\left(-8 B A D-8 C B A+4 B A^{2}+4 B^{2} A\right) v^{7} \\
& +\left(-32 C B^{2} A^{2} D+16 D^{2} A^{2} B^{2}+16 B^{2} C^{2} A^{2}\right) v^{6}
\end{aligned}
$$




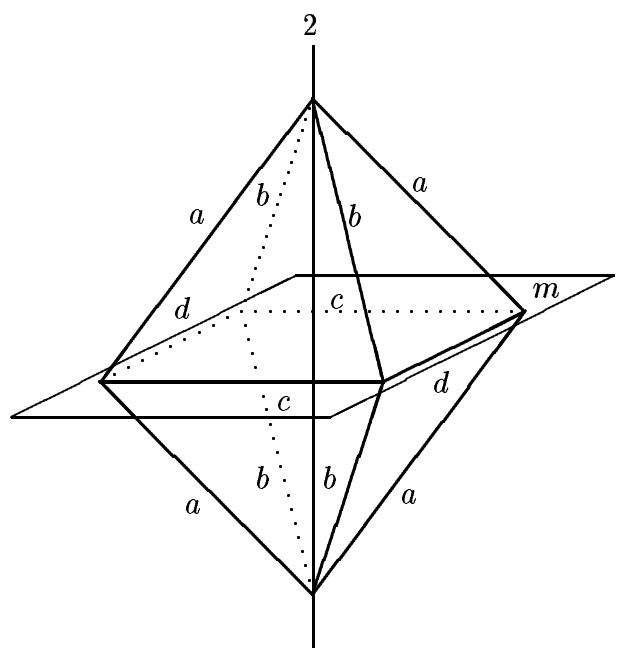

Рис. 15

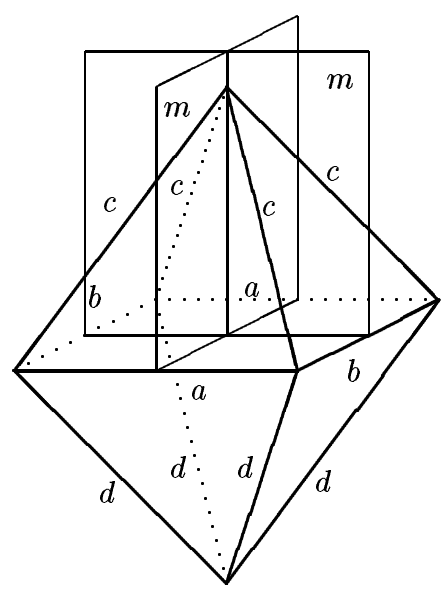

Рис. 17

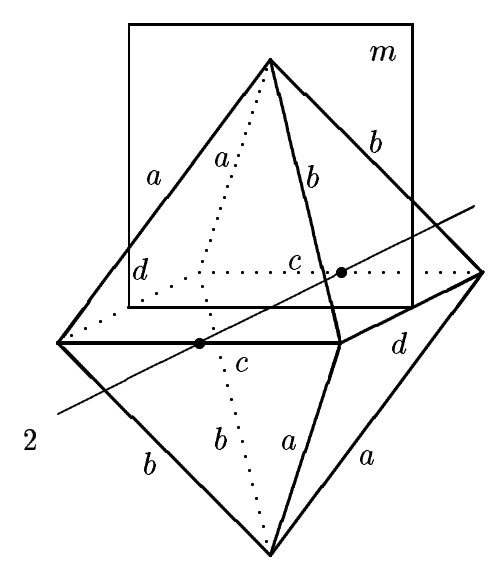

Рис. 16

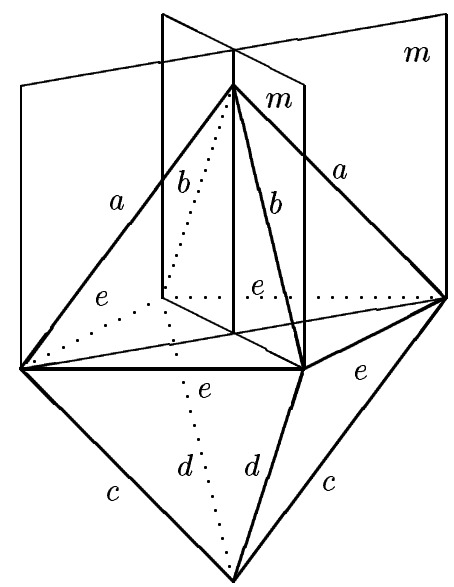

Рис. 18

18b) $m m 2$ - образующим соответствуют две координатные плоскости симметрии (см. рис. 18) и

$$
\begin{aligned}
Q(v)= & v^{8}+\left(-4 C E^{2}+4 C A^{2}-8 C A D-8 C B D+4 B^{2} D-8 E D B+4 B D^{2}\right. \\
& +4 C B^{2}-8 C B A+4 B C^{2}-8 B A D+8 B C E+8 D A E+4 A^{2} D \\
& \left.+4 A D^{2}+8 E^{3}-8 C E A-4 B E^{2}-4 D E^{2}-4 A E^{2}+4 A C^{2}\right) v^{7} \\
& +\left(-16 B C^{3} A^{2}+32 B^{2} A D^{2} E-16 B C D^{2} E^{2}-16 A C^{2} D E^{2}+32 C^{2} B A^{2} E\right. \\
& -16 C B A^{2} E^{2}+32 A^{2} C^{2} D E-64 C^{3} D A B-64 D^{3} A B C+16 D^{4} A B \\
& -16 B^{3} C^{3}+16 C^{4} B A+32 C^{3} A B^{2}-16 C B^{3} E^{2}+32 C B^{2} E^{3} \\
& -64 A^{3} D C B-64 C B^{3} A D-64 A^{2} D^{2} E B+32 A D^{2} B C E-32 A D^{3} E B \\
& -64 A^{2} D^{2} E^{2}+32 A^{2} D^{3} E-16 A D^{3} E^{2}-64 B^{2} C^{2} E^{2}-16 B C^{3} E^{2}
\end{aligned}
$$




$$
\begin{aligned}
& +32 B C^{2} E^{3}+16 C B^{4} D+32 A^{3} D^{2} E+32 A^{2} D B C E+32 B C^{2} D A E \\
& -32 A^{3} D C E-64 C^{2} B^{2} E A+32 A D^{2} E^{3}-16 A^{3} D E^{2}+32 A^{2} D E^{3} \\
& +16 A^{4} D C-64 B^{2} C^{2} E D-32 C B^{3} E D-64 A^{2} D^{2} C E+32 B^{2} C^{3} E \\
& +32 A D^{2} B E^{2}+32 A D^{2} C E^{2}+32 C^{2} B^{3} E+32 A^{2} D B E^{2}+32 A^{2} D C E^{2} \\
& +32 C B^{2} D E^{2}+32 B C^{2} D E^{2}+32 B C^{2} A E^{2}+96 A C^{2} D^{2} B+16 C D E^{4} \\
& -16 B C E^{4}-32 C B A E^{3}+96 C B^{2} A^{2} D-32 A C D E^{3}+16 A B E^{4} \\
& -16 B^{2} A D E^{2}-32 B A D E^{3}+32 B^{2} C D^{2} E-32 B C D E^{3}+32 C B^{2} A E^{2} \\
& -16 D A E^{4}+32 C B^{2} D A E+64 C^{2} D A^{2} B-80 C^{2} D B^{2} A+32 C D^{2} A^{3} \\
& -16 C D^{2} B^{3}+32 C^{2} D B^{3}-16 C^{2} D A^{3}+64 C D^{2} B^{2} A-16 D^{3} A^{3} \\
& \left.-80 C D^{2} A^{2} B+32 D^{3} A^{2} B-16 D^{3} B^{2} A-32 C^{3} A B E\right) v^{6} .
\end{aligned}
$$

19a) 222 - образующими являются координатные оси симметрии 2-го порядка 35 и 24 . Метрическая симметрия в этом случае такова:

$$
l_{23}=l_{34}=l_{45}=l_{52}=a, \quad l_{13}=l_{36}=l_{15}=l_{56}=b, \quad l_{12}=l_{26}=l_{14}=l_{46}=c,
$$

а многочлен для объема имеет следующий вид:

$$
\begin{aligned}
Q(v)= & v^{8}+\left(-8 B^{2} A+8 A^{3}+8 B^{3}-8 A C^{2}-8 C A^{2}\right. \\
& \left.+8 C^{3}+16 C B A-8 B A^{2}-8 C B^{2}-8 B C^{2}\right) v^{7} .
\end{aligned}
$$

19b) 222 - образующими являются две диагональные оси симметрии 2-го порядка: ось, проходящая через середины ребер 23,45 и ось, проходящая через середины ребер 34 , 25. Метрическая симметрия в этом случае такова:

$$
l_{34}=l_{25}=a, \quad l_{23}=l_{45}=b, \quad l_{13}=l_{15}=l_{62}=l_{64}=c, \quad l_{12}=l_{14}=l_{63}=l_{65}=d,
$$

а многочлен для объема имеет следующий вид:

$$
\begin{aligned}
Q(v)= & v^{8}+\left(-8 C B A+4 B C^{2}-8 B A D-8 C B D+4 A D^{2}+4 B D^{2}-8 C A D\right. \\
& \left.-8 C D^{2}+4 B A^{2}+8 D^{3}-8 C^{2} D+8 C^{3}+4 B^{2} A+4 A C^{2}\right) v^{7} \\
& +\left(32 B C^{3} A^{2}+128 C^{3} D A B+128 D^{3} A B C-16 C^{6}-96 D^{4} A B\right. \\
& -16 D^{6}+64 C^{3} A D^{2}+64 C^{3} B D^{2}-96 C^{4} B D-96 C^{4} A D \\
& +64 C^{2} D^{3} B+320 C^{3} D^{3}-240 D^{4} C^{2}+64 C^{2} D^{3} A-96 C D^{4} B \\
& +96 C^{5} D-96 C D^{4} A-240 C^{4} D^{2}+96 D^{5} C+32 D^{5} B+32 D^{5} A \\
& +32 C^{5} B-96 C^{4} B A+32 C^{3} A B^{2}-16 B^{2} C^{2} A^{2}-64 A C^{2} D^{2} B \\
& +32 C B^{2} A^{2} D+32 A C^{5}-32 C^{2} D A^{2} B-32 C^{2} D B^{2} A-16 D^{2} A^{2} B^{2} \\
& \left.-32 C D^{2} B^{2} A-32 C D^{2} A^{2} B+32 D^{3} A^{2} B+32 D^{3} B^{2} A\right) v^{6} .
\end{aligned}
$$

Рассмотрим оставшиеся подгрушшы порядка 4.

20) 4 - образуюшим является ось симметрии 4-го порядка, проходяшая через вершины 1 и 6. Метрическая симметрия в этом случае такова:

$$
l_{12}=l_{13}=l_{14}=l_{15}=a, \quad l_{62}=l_{63}=l_{64}=l_{65}=b, \quad l_{23}=l_{34}=l_{45}=l_{52}=c,
$$


а многочлен для объема имеет следующий вид:

$$
Q(v)=v^{8}+\left(-8 A C^{2}+8 C^{3}-8 B C^{2}\right) v^{7}+\left(16 B^{2} C^{4}-32 C^{4} B A+16 C^{4} A^{2}\right) v^{6} .
$$

21) $\overline{4}$ - образующим является инверсионная ось симметрии 4-го порядка, проходящая через вершины 1 и 6 . Метрическая симметрия в этом случае такова:

$$
l_{13}=l_{15}=l_{62}=l_{64}=a, \quad l_{12}=l_{14}=l_{63}=l_{65}=b, \quad l_{23}=l_{34}=l_{45}=l_{52}=c,
$$

а многочлен для объема имеет следующий вид:

$$
\begin{aligned}
Q(v)= & v^{8}+\left(-16 C B A-8 B C^{2}+8 B^{3}-8 B A^{2}+8 C A^{2}\right. \\
& \left.+8 C B^{2}+8 C^{3}-8 B^{2} A-8 A C^{2}+8 A^{3}\right) v^{7} \\
& +\left(128 B^{3} C A^{2}-64 B C^{3} A^{2}-16 A^{6}-192 B A^{4} C+128 A^{3} B^{2} C\right. \\
& +64 A^{3} C^{3}-240 A^{4} B^{2}+96 A^{5} B-96 A^{4} C^{2}+64 A^{5} C \\
& +128 B A^{3} C^{2}+320 A^{3} B^{3}-16 B^{6}-16 B^{2} C^{4}+64 B^{3} C^{3} \\
& -16 C^{4} A^{2}+64 C B^{5}-240 A^{2} B^{4}+32 C^{4} B A-192 C B^{4} A \\
& \left.-64 C^{3} A B^{2}+128 C^{2} B^{3} A-96 B^{4} C^{2}-64 B^{2} C^{2} A^{2}+96 A B^{5}\right) v^{6} .
\end{aligned}
$$

Подгруппа порядка 3 порождается осью симметрии третьего порядка.

22) 3 - образующим является ось симметрии 3-го порядка (проходящая через центры граней 134 и 256). Метрическая симметрия в этом случае такова:

$$
l_{13}=l_{34}=l_{13}=a, \quad l_{62}=l_{65}=l_{25}=b, \quad l_{12}=l_{45}=l_{63}=c, \quad l_{15}=l_{23}=l_{64}=d,
$$

а многочлен $Q(V)$ содержит 7781 моном, поэтому выписьвать его здесь не имеет смысла.

Следующие подгруппы имеют порядок $2: i, 2, m$.

23) $i$ - подгруппа порождена отражением относительно центра симметрии; метрически этот октаэдр устроен как изгибаемьй октаэдр Брикара 1-го типа (см. рис. 19) и

$$
\begin{aligned}
Q(v)= & v^{8}+(-4 B A D+4 F C A-4 E A B-4 F E D-4 E F A \\
& -4 C B D-4 C B A-4 E F B-4 E F C-4 C A D-4 C E D \\
& +4 E F^{2}+4 C^{2} D+4 D A E-4 F A B+4 F E^{2}+4 D F B \\
& \left.+4 B A^{2}+4 B C E-4 D F C+4 C D^{2}+4 B^{2} A\right) v^{7} .
\end{aligned}
$$

24а) 2 - образующим является координатная ось симметрии 2-го порядка, проходящая через вершины 1 и 6; метрически этот октаэдр устроен как изгибаемьй октаэдр Брикара 2-го типа (см. рис. 20) и

$$
\begin{aligned}
Q(v)= & v^{8}+\left(4 C E A-4 B A D-4 F A B-4 E A B+4 B^{2} A-4 B F C\right. \\
& +4 D E^{2}+4 C E^{2}-8 F E D-8 C E D+4 F D^{2}-4 F C A \\
& +4 E C^{2}+4 D F^{2}-8 D F C-8 E F C+4 D F B-4 C B A+4 B A^{2} \\
& \left.+4 D F A+4 B C E-4 D A E-4 E D B+4 E D^{2}+4 F^{2} C+4 F C^{2}\right) v^{7} \\
& +\left(16 B C D^{2} E^{2}-32 A C^{2} D E^{2}+16 C^{2} B A^{2} E+16 C B A^{2} E^{2}-80 C^{2} E^{2} D F\right. \\
& -80 D^{2} F^{2} C E+16 C E^{2} D F A-64 C E^{3} F D+96 F^{2} E^{2} D C+16 E C^{2} D F B
\end{aligned}
$$




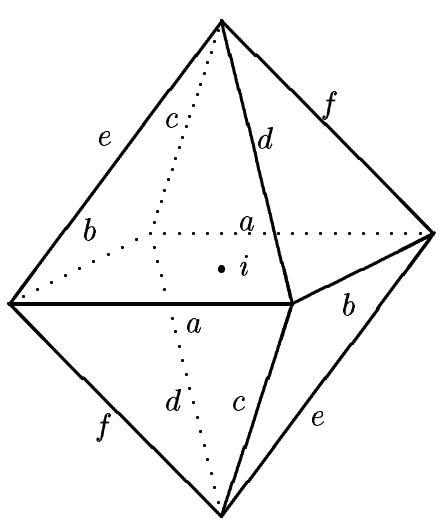

Рис. 19

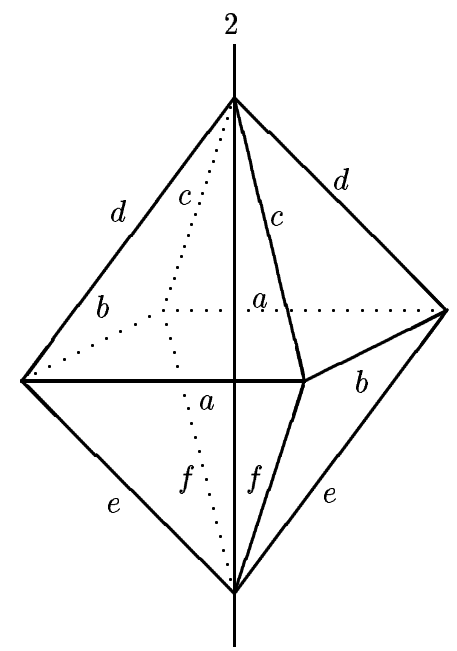

Рис. 20

$$
\begin{aligned}
& +16 E C^{2} D F A-16 D F C B A^{2}-64 E C^{3} D F+16 E F^{2} C^{2} B-16 E C^{3} B F \\
& -16 E C^{3} F A+64 F E^{2} D^{2} C+16 E F^{2} C^{2} A+16 D F^{2} B C E+16 D F^{2} C E A \\
& +16 D F^{2} C^{2} B-64 D F^{3} E C+16 D F^{2} A B^{2}+64 D F^{2} C^{2} E+16 F E^{2} D^{2} B \\
& +16 F E^{2} D^{2} A-16 E A^{2} B F C-16 D F^{3} A B-16 E A B^{2} F C+16 C E^{2} D F B \\
& +16 D F^{2} C^{2} A-16 D F^{3} C A-16 E^{3} C^{3}-32 C^{2} E^{2} F A-32 C^{2} E^{2} B F \\
& +96 D^{2} F C^{2} E-32 D^{2} F^{2} E B-32 D^{2} F^{2} A E-32 F^{2} D^{2} C A-16 D F^{3} B C \\
& +16 F D^{2} B C E-64 F D^{3} C E+16 F D^{2} C E A-16 D F C A B^{2}-16 F D^{3} E B \\
& -16 F D^{3} A E-32 F^{2} D^{2} B C+16 B^{2} A D^{2} F-16 B A D^{3} F+16 D F^{2} B A^{2} \\
& +16 B A^{2} D^{2} F-16 E A B^{2} D F-16 E A^{2} B D F-16 A D^{2} B C E+16 B C^{3} E^{2} \\
& +16 B C^{2} E^{3}-16 A^{2} D B C E+32 B C^{2} D A E+16 C^{2} B^{2} E A+16 A D^{2} C E^{2} \\
& -32 B C^{2} D E^{2}-64 B C^{2} A E^{2}+16 C D E^{4}-16 C B A E^{3}+16 C B^{2} A^{2} D \\
& -16 A C D E^{3}-16 B C D E^{3}+16 C B^{2} A E^{2}-16 E A^{2} C B^{2}-16 C B^{2} D A E \\
& -16 E A B F^{2} C+32 E A B F^{2} D+32 E^{2} A B F C-16 E^{2} A B F D+32 E A B F D^{2} \\
& +32 E A B F C^{2}+16 F D^{4} E+16 D^{2} F^{3} A+16 E A^{2} F B^{2}+16 D^{2} F^{3} B \\
& +16 D F^{4} C-16 B A D F C^{2}+32 B A D^{2} F C+32 B A D F^{2} C-64 B A D^{2} F^{2} \\
& -16 A^{2} D F B^{2}+16 E C^{4} F+32 E^{2} A B C D+16 E^{2} C^{3} A+16 C^{2} E^{3} A \\
& +32 F^{2} D^{3} E+32 C^{2} E^{3} D-16 E F^{2} C^{3}+16 F^{2} D^{3} A \\
& +16 F^{2} D^{3} B-16 F D^{3} E^{2}+32 C^{3} E^{2} F-16 D F^{3} C^{2} \\
& \left.-16 C E^{3} D^{2}+32 D^{2} F^{3} C-16 C^{3} A B E-16 D^{3} F^{3}\right) v^{6} .
\end{aligned}
$$

Следующие случаи приведены лишь для полноты изложения: полином в каждом из этих случаев получается, по-видимому, весьма сложным, и вычислить его в общем виде не удалось.

24b) 2 - образующим является диагональная ось симметрии 2-го порядка, проходя- 
щая через середины ребер 23 и 45. Метрическая симметрия в этом случае такова:

$$
\begin{gathered}
l_{12}=l_{63}=a, \quad l_{13}=l_{62}=b, \quad l_{15}=l_{64}=c, \quad l_{14}=l_{65}=d, \\
l_{34}=l_{25}=e, \quad l_{23}=f, \quad l_{45}=g .
\end{gathered}
$$

25a) $m$ - образующим является диагональная плоскость симметрии, проходящая через вершины 1 и 6 и середины ребер 25 и 34 . Метрическая симметрия в этом случае такова:

$$
\begin{gathered}
l_{13}=l_{14}=a, \quad l_{63}=l_{64}=b, \quad l_{62}=l_{65}=c, \quad l_{12}=l_{15}=d, \\
l_{23}=l_{45}=e, \quad l_{34}=f, \quad l_{25}=g .
\end{gathered}
$$

25b) $m$ - образующим является координатная плоскость симметрии, проходящая через вершины $2,3,4,5$. Метрическая симметрия в этом случае такова:

$$
\begin{gathered}
l_{12}=l_{62}=a, \quad l_{13}=l_{63}=b, \quad l_{14}=l_{64}=c, \quad l_{15}=l_{65}=d, \\
l_{23}=e, \quad l_{34}=f, \quad l_{45}=h, \quad l_{52}=g .
\end{gathered}
$$

\section{СПИСОК ЦИТИРОВАННОЙ ЛИТЕРАТУРЫ}

[1] Сабитов И. Х. Обобщенная формула Герона-Тарталья и некоторые ее следствия // Матем. сб. 1998. Т. 189. №10. С. 105-134.

[2] Астрелин А.В., Сабитов И.Х. Минимальный по степени многочлен для объема октаэдра // УМН. 1995. Т. 50. № 5. С. 245-246.

[3] Галиулин Р. В. Лекции по геометрическим основам кристаллографиии. Челябинск: Изд-во Челябинского университета, 1989.

Московский государственный университет им. М.В. Ломоносова E-mail: mihalyov@mtu-net.ru 